Max Claasz in Rostock i. M.: Ein Verfatren zur Herstellung einer Verbindung aus Rindermile mit Eisen. D.R.P. 122492 vom 24. Maj 1899. - Patentbl. 1901, $22,1177$.

Zerkleinerte Rindermilz wird mit etwa $10^{\circ}$ o-igem Alkohol ausgelaugt, das Filtrat in eine verdünnte Eisenchloridlosung hineingegossen, die Mischung mittelst verdinnter Natronlauge ausgefällt, der Niederschlag getrocknet und unter Umständen gepulvert.

Chemische Fabrik von Heyden, Aktien-Gesellschaft in Radebeul bei Dresden: Vexfahren zur Darstellung von albumose-und aschefreiem Pepton. D.R.P. 122167 vom 8. August 1900. - Patentbl. 1901, 22, 1185.

Rohpeptonlösungen werden mit Ammoniumsulfat, Zinksulfat oder einem anderen albumosefällenden Mittel gesättigt, worauf mittelst koncenrtrirter Schwefelsäure und Ferrivelzen die Ietzten Albumosereste entfernt werden. Aus dem Filtrat wird hierauf durch Ferribalze unter Abstumpfen der Säure ein Eisenpeptonniederschlag ausgefällt. Letzterer wird abfiltrirt, mit einer gesättigten Lösung von Ammoniumsulfat gewaschen und unter Zusatz von etwas Ammoniak in Wasser verrührt. Durch Baryumbydroxyd wird dss Eisen und die Schwefelsäure abgeschieden und im Filtrat der überschüssige Baryt durch Kohlensäure gefällt. Das Filtrat wird im Vakuum eingedampft, der Rückstand in Essigsäure gelöst und durch Alkohol gefällt.

Fried. Jos. Freih. von Mehxing in Halle a. S.: Verfahrenzur Hergtellung oines Nährmittels bezw. Nährmehles aus Magermileh und Eidotter ovent. unter Zusatz von Koblenhydraien. D.R.P 121907 vom 28. September 1899. Patentbl. 1901, 22, 1121.

Bekanntlich ist das Eidotterfett selbst bei jahrelanger Aufbewahrung und dementsprechend auch der Eidotter in getrocknetem Zustande unverändert haltbar; ebenso bleibt der urspringliche Geschmack unverändert erhalten. Diese Eigenschaften fehlen den meisten Pflanzenfetten, sowie ganz besonders dem Butterfett. Unter Zugrundelegung dieser Erfahrung wird ein besonders für Kinder geeignetes Nährmittel dadurch hergestellt, dass man das Milchfett durch das Eidotterfett ersetzt und dieses mit Magermileh und Eidotter eintrocknet. Dem Gemisch kann man dann noch je nach Bedarf Kohlenhydrate, wie Mehl und Zucker und dgl. zusetzen.

Jean Peeters in Schaerbeck: Verfahren zur Gewinnung von Albumosen, Peptonen und anderen stickstoffhaltigen Körpern aus Hefe. D.RP. 124985 vom 17. April 1898. - Patentbl. 1901, 22, 1560.

Die Hefe wird mit einer 0,2 bis $0,5 \%$-igen wässerigen Weinsäurelösung bei einer Temperatur von 45 bis $50^{\circ}$ behandelt. Die Behandlung mit Weinsäure hat vor der bereits bekannten, dem gleichen Zweck dienenden Behandlung mit Salzsäure oder Milchsäure den Vortheil, dass dabei ein grosser Theil der in der Hefe vorhandenen Kalisalze als Bitartrate zur Ausscheidung gelangt und durch Filtration abgeschieden werden kann, bezw, von dem durch Eindampfen gewonnenen Extrakte durch nochmaliges Aufiosen in Wasser und nochmaliges Filtriren getrennt werden kann. Die erbsltenen Produkte haben ausserdem einen angenehmen Geschmack.

A. Oelker.

\title{
Tabak.
}

T. E. Thorpe und J. Holmes: Ueber das Vorkommen von Paraffinen im Tabaksblatt. - Proceedings Chem. Soc. 1901, 17, 170-171.

Bei der Untersuchung eines mittels Petroläther gewonnenen Extraktes entdeckten Verff. in demselben zwei Kohlenwasserstoffe, das Hentriacontan $\left(\mathrm{C}_{\mathbf{3 1}} \mathbf{H}_{64}\right)$ vom Schmelzp. 67,8-68,5 und das Heptacosan $\left(\mathrm{C}_{27} \mathrm{H}_{56}\right)$ vom Schmelzp. 59,3-59,8. Dieselben sind im Tabaksblatt ungefähr-in gleichen Mengen vorhanden und zwar bis zu $1 / 10 \%$.

Den Schmelzpunkt der aus Kentucky- und Virginia-Tabaksblättern gewonnenen Mischungen oben genannter Kohlenwasserstoffe fanden Verff. bei drei aus vensehie- 
denen Theilen der Blätter gewonnenen Präparaten zu 63,0-63,8 63,5-64 und 63,7 bis $65^{\circ}$. Sie folgern hieraus, dass die weisse Substanz vom Schmelzp. $63^{\circ}$, welche von Kissling aus Kentucky-Tabak extrahirt wurde (Ber. Deutsch. Chem. Ges. 1883, 16, 2432) and welche er für einen unreinen Melissinsäuremellisylester hielt, sowie die von eben demselben aus Tabakrauch isolirte Substanz vom Schmelzpunkte $64,5^{\circ}$, die aber von ihm als ein Koblenwasserstoff angesehen wurde, in Wirklichkeit dieselben Produkte und zwar ein Gemisch der oben genannten Paraffine, Hentriacontan und Heptacosan darstellen.

A. Oelker.

Richard Kissling: Ueber das Vorkommen von Paraffinen im Tabakblatt. - Chem.-Ztg. 1901, 25, 684 .

Thorpe und $\mathrm{Holmes}$ berichten in einer der Chemical Society vorgelegten Arbeit (Vergl. das vorstehende Referat!), dass sie im Petroläther-Extrakte des Tabakblattes die Gegenwart zweier Paraffine, des Hentriakontans, $\mathrm{C}_{31} \mathrm{H}_{64}$, und des Heptakosans, $\mathrm{C}_{27} \mathrm{H}_{56}$, nachgewiesen haben, und dass sie die vom Verf. früher aus dem Tabak abgeschiedene wachsartige Substanz für ein Gemisch dieser beiden Paraffine halten. Verf. glaubt nicht, dass in der lebenden Pflanze Paraffine gebildet werden, und hält die von ihm aus dem Tabak isolirte und analysirte Substanz nach wie vor für eine Wachsart, deren Zusammensetzung der des Melissinsäure-Melissylesters nahekommt.

\section{A. Hebebrand.}

Oskar Loew: Nochmals über die Tabakfermentation. II. - Centralbl. Bakteriol. II. Abth. 1901, 7, 673-680.

Die Fermentation des Tabaks vollzieht sich nach dem Verf. durch die Wirkung von oxydirenden Fermenten, nach Behrens und anderen Forschern ist sie eine Folge der Bakterienthätigkeit. Der Verf. wendet sich gegen die letzte Veröffentlichung von Behrens (Diese Zeitschrift 1901, 4, 1041) und entkräftet dessen „Widerlegung". Zunächst weist der Verf. aus seinen Schriften nach, dass er die Tödtungstemperatur der Oxydase nicht als eine unter allen Bedingungen konstante Grösse betrachte. Ein dachreifer Tabak, welcher seine Oxydase verloren hat, kann immer noch das widerstandsfähigere Zymogen derselben besitzen, welches später zur Wirkung gelangen könnte. Hierfür sprechen verschiedene Beobachtungen. Die Behauptung von Behrens, dass Nikotinsalze weder durch Oxydase noch durch Peroxydase angegriffen werden, stimmt nicht mit den Beobachtungen des Verf.'s, nach welchen bei dieser Einwirkung Spuren Ammoniak entstehen. Dass Nikotinsalze von den Bakterien als Nahrung benutzt werden können, hat der Verf. bereits früher beobachtet.

Dass Bakterien nicht die Ursache der Tabakfermentation sind, geht aus der folgenden Thatsache hervor: Wenn in einem Tabakhaufen die Temperatur in Folge der Fermentation $50-60^{\circ}$ erreicht hat, dann werden die Haufen auseinandergenommen, um schädliche Wirkungen einer noch höheren Temperatur zu vermeiden. Ein Theil der oxydirenden Enzyme ist dabei zerstört worden, weshalb beim Wiederaufbau der Tabakhaufen statt 4 Tage es nun etwa 6 Tage dauert, bis dieselbe Temperaturhöhe erreicht ist. Wären Bakterien die Ursache der Tabakfermentation, so müssten sie sich während ihrer Thätigkeit vermehrt haben und die Temperatursteigerung müsste nun bein Wiederaufbau der Haufen um so rascher erfolgen.

Für die Behauptung, dass Bakterien noch auf Tabak mit $25 \%$ Wassergehalt gedeihen können, hat Behrens den Beweis nicht geliefert. Gegen die bezügliehen 
Versuche ist Manches einzuwenden, besonders, dass es unmöglich ist, einen zerkleinerten Tabak gleichmässig mit der angegebenen Wassermenge zu mischen.

A. Hebebrand.

J. Habermann: Beiträge zur Kenntniss des Cigarrenrauches. - Zeitschrift physiol. Chem. 1901, 33, 55-125.

Ohne Berücksichtigung der neueren Arbeiten über die Bestandtheile des Cigarrenrauches (Thoms, diese Zeitschr. 1899, 2, 798; Wahl. diese Zeitschr. 1900, 3, 561) theilt Verf. in einer sehr umfangreichen Veröffentlichung die Ergebnisse seiner mit 10 österreichischen Regie-Cigarren ausgeführten Untersuchungen mit. Die Nikotinbestimmung wurde nach der alten Methode von $\mathrm{Kissling} \mathrm{(Zeitschr.} \mathrm{analyt.} \mathrm{Chem.}$ 1882, 21, 64), aber unter Verwendung von Methylorange als Indikator ausgeführt. Die 10 untersuchten österreichischen Cigarrensorten enthielten 16,4 bis 22,1\% Asche, 5,64 bis $7,27 \%$ Feuchtigkeit und 1,29 bis $3,99 \%$ Nikotin.

Die Rauchversuche wurden in der Weise ausgeführt, dass sie dem natürlichen Verlaufe des Cigarrenrauchens entsprachen; der Rauch wurde nicht gleichmãseig abgesogen, sondern intermittirend mit Hilfe eines vom Verf. konstruirten Aspirators. Als Absorptionsmittel für die Rauchprodukte dienten Baumwolle, Bleibaumwolle (getränkt mit Bleizucker- und Natriumkarbonatlōsungen), Kalilauge oder Natronlauge, verdünnte Schwefelsäure. Die Rauchversuche hatten die nachstehenden Ergebnisse, welche theils im Finklang, theils aber anch im Gegensatze zu denen anderer Forscher stehen: Im Rauch aller untersuchten Cigarrensorten wurden Schwefelwasserstoff und Kohlenoxyd nachgewiesen, Blausäure aber nicht. Die Kohlenoxydmengen schwankten für $1 \mathrm{~g}$ verrauchter Cigarre zwischen 5,2 und $19,3 \mathrm{ccm}$. Im Durchschnitt der Resultate särnmtlicher Rauchversuche ist die Menge der entwickelten Kohlensäure viermal so gross als die Menge des Kohlenoxyds, doch zeigten einige Sorten erhebliche Abweichungen von diesem Verhältniss. Es scheint, als ob dasselbe abhängig ist von der Qualität des Tabaks und der Energie des Rauchens. Eine mittlere Cigarre im Gewicht von 5,5 g entwickelt im Durchschnitt 3,5 Liter Rauch. Bezüglich der Schicksale des Nikotins beim Verrauchen wurde ermittelt, dass $3,6 \%$ in den abgeschnittenen Spitzen, $36,2 \%$ in den unverrauchten Stummeln, 16,3\% $\mathrm{m}$ angesaugten Rauche und $44 \%$ theils im nicht angesaugten Rauche verbleiben, theils zerstört werden. Es kann aber keinem Zweifel unterliegen, dass eine erhebliche Menge der in Rauche aufgefundenen Stickstoffbasen aus Pyridinbasen besteht, von denen ein Theil dem beim Rauchen zerstörten Nikotin, der grössere Theil aber wahrscheinlich dem Eiweiss des Tabaks entstammt. Als Hauptergebniss der Arbeit des Verf.'s ist herrorzuheben, dass nur ein verhältnissmässig kleiner Theil des Nikotins in den angesaugten Rauch übergeht.

A. Bebebrand.

\section{Gesetze, Gesetz - Entwürfe, Verordnungen u. s. w., Gerichts - Fntscheidungen.}

\section{Allgemeines.}

Preussen. Bekanutmachung des Ministers der geistlichen, Unterrichtg- und Medicinal-Angelegenheiten vom 16. Mai 1902 betr. die Präfungskommissionen für 